\title{
Controller Performance Assessment of a Photovoltaic Generator Terminated in a Current- Mode-Buck-Convertor-Load
}

\author{
Moshe Sitbon $^{1}$, Martin Mellincovsky ${ }^{2}$, Ilan Aharon ${ }^{1}$ \\ ${ }^{1}$ Department of Electrical Engineering and Electronics, Ariel University, \\ Ariel, Israel \\ ${ }^{2}$ Department of Electrical and Electronics Engineering, Shamoon College of Engineering, \\ Ashdod, Israel \\ moshesi@ariel.ac.il
}

\begin{abstract}
In this paper, a comprehensive control analysis is presented, with emphasis on regulating the terminal voltage of a photovoltaic generator, interfaced with a current mode controlled buck DC-DC converter, which is, in turn terminated by a battery. The combined generator-convertor-load system possesses nonlinear behaviour, and is subject to environmental conditions, thus causing the control task complicated. Additionally, analyses of small signal equations reveal a possible unstable condition. Hence, a new method of designing a controller for worst-case scenario is presented. Additionally, results of implementing a typical linear controller (PI), which can be designed only in reference to a single nominal operation point, revealing a varied responses. Simulation and results of mathematical analysis are presented to verify the proposed method.
\end{abstract}

Index Terms-Control design; DC-DC power converters; Mathematical analysis; Photovoltaic systems.

\section{INTRODUCTION}

Photovoltaic generator (PVG) based systems are mainly designed to extract the maximum attainable energy from their solar source [1], utilizing diverse of maximum power point tracking (MPPT) algorithms [2], [3]. Nonetheless, following recent developments in microgrids concept [4], [5], renewable energy generators are now required to operate below the maximum power point (MPP). In addition, they need to match generation and consumption levels in isolated microgrids [6]-[8]. As a result, the supervising power management controller (PMC) should be able to operate in both modes. PMC-generated output may serve as PVG voltage or current reference in addition to the interfacing converter duty cycle. Quantitative research [9][14] has indicated that controlling PVG voltage is the best option due to higher stability and lower dependence on solar irradiation.

However, operation below MPP may require wide PVG voltage variation as opposed to the MPPT mode, where PVG voltage range is relatively narrow [15]. Recently, it was shown that source properties significantly influence power converter dynamics [16]. PVG dynamic resistance is highly dependent on the operating point and may experience

Manuscript received 11 November, 2018; accepted 9 March, 2019. major variation. Hence, the combined PVG-converter-load system dynamics undergo significant changes.

These deviations are caused by PVG operation point variations and directly influence the system, from damping up to losing stability [17].

Voltage control structures often utilize an inner current loop, which, in addition to inherent overcurrent protection, enhances robustness and reduces system order, simplifying the controller design. PVG is typically integrated with a boost power stage. In this paper, this is accomplished by sweeping into the "buck" convertor, which leads to the cascaded control shown in Fig. 1.

The controllers are usually decoupled in terms of bandwidth to allow proper independence of loops and ease of control design.

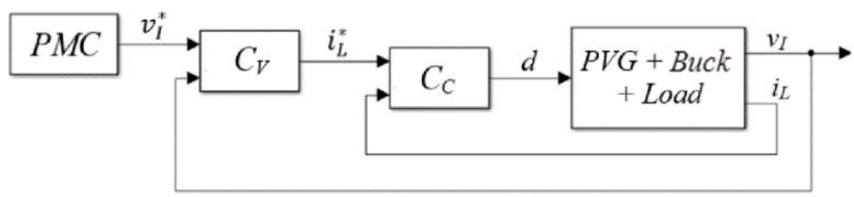

Fig. 1. Cascaded control structure under investigation.

As shown in [18], [19], the voltage loop small-signal dynamics must be contingent on PVG as well as on load properties. Nevertheless, since load properties in PVG-based systems are slow-varying, the source influence is far more significant. Conventionally, PVGs were terminated by large capacitors and represented by ideal current sources in control design development. In this paper, A novel mathematical analysis approach for PVG-Buck-Load transfer function is presented. The new method employs variables that allows rewrite the system transfer function and simplify it. The new terms enable a bridge system transfer function into a form that assist to set the nominal plant without entering to an unstable zone due the existence of the right hand pole in buck converter. In addition, the new method allows us to reveal the system behaviour from main components perspective and enable us to present control performance analysis under a range of conditions and constrains. To verify the new method a deep analysis is made, then setting the system behaviour by preferring controller variables and then affirming the proposed theory 
by simulation.

\section{PROBLEM FORMULATION}

The system under consideration is shown in Fig. 2, in which the PVG capacitor is interfaced with buck power stage (BuPS). The converter is integrated to a battery (in the case of autonomous systems) or coupled to the grid by a voltage control inverter. In both methods, the converter load is of a voltage-source type and may commonly be represented by a Thevenin equivalent circuit with slowvarying parameters.

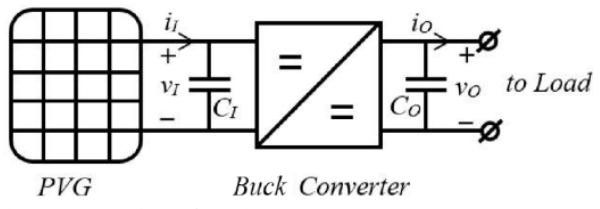

Fig. 2. Buck converter interfaced PVG.

The resultant average model of the combined PVG-BuPSLoad system is shown in Fig. 3. $\mathrm{L}$ and d represent converter inductance and duty cycle, respectively, $R_{B}$ and $V_{B}$ denote load Thevenin parameters, and the PVG is built from current source and a paralell resistor as a Norton equivalent circuits in the electrical diagram of solar cell, when $R_{P V}$ reflects $P V$ static resistance at MPP.

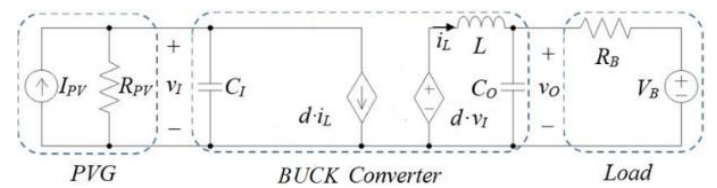

Fig. 3. Average model of combined PVG-Buck Converter-Load system.

The combined system is governed by the following set of equations, assuming a continuous conduction mode:

$$
\left\{\begin{array}{l}
C_{I} \dot{\tilde{v}}_{I}=-R_{P V}^{-1} \tilde{v}_{I}-I_{L} \tilde{d}-D \tilde{i}_{L}, \\
\dot{\tilde{i}}_{L}=D \tilde{v}_{I}+V_{I} d-\tilde{v}_{O}, \\
C_{O} \dot{\tilde{v}}_{O}=\tilde{i}_{L}-R_{B A T}^{-1} \tilde{v}_{O},
\end{array}\right.
$$

linearized around an operation point given by:

$$
\left\{\begin{array}{l}
\frac{P_{P V}}{V_{I}}=I_{P V}-V_{I} R_{P V}^{-1}=D I_{L}, \\
D V_{I}=V_{O}, \\
\frac{P_{P V}}{V_{O}}=I_{L}=\left(V_{O}-V_{B A T}\right) R_{B A T}^{-1}
\end{array}\right.
$$

As internal battery resistance $\left(R_{B A T}\right)$ is small-scale in size, zero value can be assumed. And as $V_{O}$ can be declared constant due to battery characteristics, this leads to the assumption that the small signal of $V_{O}$ has no effect on internal voltage [20]. Consequently, the next set of equations is as follows:

$$
\left\{\begin{array}{l}
C_{I} \dot{\tilde{v}}_{I}=-R_{P V}^{-1} \tilde{v}_{I}-I_{L} \tilde{d}-D \tilde{i_{L}}, \\
L \dot{\tilde{i}}_{L}=D \tilde{v}_{I}+V_{I} d
\end{array}\right.
$$

Taking into account the cascaded control structure of Fig. 1 and with the conditions that the current loop is properly closed and its bandwidth is considerably higher than the voltage loop bandwidth, perfect current tracking may be presumed

$$
\tilde{i}_{L}(t)=\tilde{i}_{L}^{*}(t)
$$

Substitution of (4) into (3) implements Laplace transform and rearranges the results, yielding

$$
\frac{\tilde{v}_{i}}{\tilde{i}_{L}^{*}}(s)=-\frac{D+\frac{I_{L}}{V_{I}} L s}{C_{i} s+R_{P V}^{-1}-R_{s}^{-1}},
$$

where

$$
R_{s}^{-1}=\frac{I_{L}}{V_{I}} D
$$

Due to the influence of $R_{P V}$ and $R_{S}$ on the transfer function (5) and throughout the change of PV voltage values, an unstable situation can occur. It is expressed when the system operates lower than MPP on the PV curve, as in Fig. 4. Thus, caution in design is necessary, in case the load is a Li-ion battery with minimum and maximum voltages that must be constrained within nominal operating values. Therefore, varying regions of PV operated voltage may be compelled, leading to different responses. Consequently, the system response is no longer as designed. In practice, it can change from highly overdamped to under-damped up to system instability and oscillation. To overcome the complication of controlling the full range of operation, a worst-case design is necessary. Accordingly, a reshaping transfer function is required.

It is noteworthy that output voltage should be restricted to a certain maximum value when the load is a battery or stand-alone system. As a result, this condition directly influences the input voltage, and it is important to note that input voltage dynamics are highly dependent on both environmental conditions and the operating point. Traditionally, designers ignored these influences by using a large capacitor. However, when using an autonomous system such as a lithium battery as a load, the internal dynamics highly influence the performance of the input voltage. Consider a representative nominal I-V curve of a PVG for certain values of irradiation $G_{O}$ and temperature $T_{\mathrm{O}}$, as shown in Fig. 4 . Note that PVG behaves as a current source at voltages lower than MPP, and as a voltage source at voltages higher than MPP. That is, it maintains high dynamic resistance in the former case and low dynamic resistance in the latter. In order to compensate for the input voltage loop, the PI controller should be of the form

$$
C(s)=-\frac{\omega_{c}}{s}\left(P_{N}^{-1}\right) .
$$

As depicted in [1] where the boost converter is regulated, it cannot be employed. Thus $\omega_{c}$ is the preferred crossover 
frequency, in order to create the "ideal" loop gain $\omega_{c} / s$. However, attaining "ideal" loop gain is a nontrivial task, since $R_{P V}$ and $R_{S}$ vary considerably and directly impact the denominator of the transfer function (5). Due to significant change in $R_{S}$ value, a positive pole can result in an unstable system (8)

$$
\left(C_{i} s+R_{P V}^{-1}-R_{s}^{-1}\right) \Rightarrow\left(R_{P V}^{-1}-R_{s}^{-1}\right)<0 .
$$

In order to solve this problem, a PI controller should be designed for the worst-case scenario $\left(v_{p v}^{\min }\right)$ as determined in Fig. (4).

Analysing regions of operation and determining the $v_{p v}^{\min }$ or $v_{p v}^{\max }$ (best-case) is required. Thus, some manipulations in (5) are necessary as the goal is to separate the gains to determine denominator influence on stability. Rearranging (5) obtains the transfer function (9). As a result, two separate parts are obtained: gain $(\mathrm{G})$ and system.

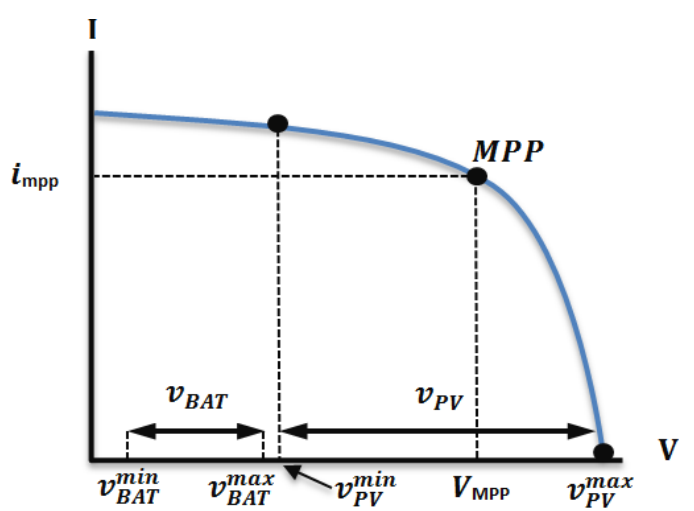

Fig. 4. $v_{p v}$ operation area.

$$
P_{(\mathrm{s})}=\frac{\tilde{v}_{i}}{\tilde{i}_{L}^{*}}=-\frac{D\left(1+\frac{L I_{L}}{V_{I} D} s\right)}{\left(R_{P V}^{-1}-R_{S}^{-1}\right)\left(1+\frac{C_{i}}{R_{P V}^{-1}-R_{S}^{-1}} s\right)} .
$$

Hsere the gain is

$$
G=\frac{D}{\left(R_{P V}^{-1}-R_{s}^{-1}\right)} .
$$

And for the remainder of the equation, a few assumptions are made. As lower frequencies were $\omega \gg \omega_{o}$ the numerator approaches one

$$
1+\frac{L I_{L}}{V_{I} D} s \rightarrow \approx 1 .
$$

And for more simplicity, $\omega_{p v}$ is defined as

$$
\omega_{p v}=\frac{R_{P V}^{-1}-R_{s}^{-1}}{C_{i}},
$$

eventually resulting in

$$
P_{(\mathrm{r})}=\frac{\tilde{v}_{i}}{\tilde{i}_{L}^{*}}=\approx-\frac{G}{1+\frac{s}{\omega_{p v}}} .
$$

The next design stage is the determination of $v_{p v}^{\min }$ or $v_{B A T}^{\max }$ limitations. Since the load is a Li-ion battery, therefore, the voltage is limited to a maximum permissible. Consequently, determined as $v_{B A T}^{\max }$, and respectively to Fig. 4, leading to $v_{p v}^{\min }$ as the worst-case scenario:

$$
\left\{\begin{array}{l}
W c: \frac{v_{B A T}^{\min }}{v_{\min }}<D_{w c}<\frac{v_{B A T}^{\max }}{v_{\min }}, \\
R_{p v}>R_{s} \Rightarrow R_{P V}^{-1}<R_{s}^{-1}, R_{s}=\frac{v_{\min }}{I_{s c}}, \\
\text { when_ } R_{p v} \rightarrow \infty \Rightarrow \omega_{p v_{-} \mathrm{wc}}=-\frac{1}{R_{s} C_{i}} .
\end{array}\right.
$$

The worst-case $\left(v_{p v}^{\min }\right)$ scenario is when the PV operates at the left side of the i-v curve, as in Fig. 4, where the duty cycle is limited between $v_{B A T}^{\min }$ to $v_{B A T}^{\max }$ with respect to $v_{p v}$. Accordingly, $\omega_{p v_{-} w c}$ is dependent on the $R_{s}$ value (14). It should be noted that the minus sign in (14) can in uncorrected design easily lead to instability.

On the other hand, when PV voltage is approximately MPP or higher, the system from the control perspective is functioning in the best-case zone. Hence, $v_{p v}$ is bonded between min and max PV operation voltages. That is, it is determined and limited by the upper threshold battery voltage due to internal protection of the battery or a safety reason ( $v_{p v}^{\min }$ scenario). The duty cycle (D) in the buck convertor is the ratio between $v_{\text {out }}$ to $v_{\text {in }}$ (15). Therefore, $v_{\max }$ should be $v_{o c}$ (PV open circuit voltage) as indicated by best-case $(\mathrm{Bc})$ voltage $\left(v_{p v}^{\max }\right)$. It should be emphasized that the result in (15) means $\omega_{p v}$ will cause a slower response time:

$$
\left\{\begin{array}{l}
v_{B A T}^{\min }<v_{o}<v_{B A T}^{\max }, \\
B c: \mathrm{D}=\frac{v_{o}}{v_{I}}, \frac{v_{B A T}^{\min }}{v_{o c}}<D_{B c}<\frac{v_{B A T}^{\max }}{v_{o c}}, \\
R_{p v}<R_{s} \Rightarrow R_{P V}^{-1}>R_{s}^{-1}, \\
\text { when_R } R_{s} \rightarrow \infty \Rightarrow \omega_{p v_{-} \mathrm{Bc}}=\frac{1}{R_{P V} C_{i}} .
\end{array}\right.
$$

A typical approach to compensation (13) utilizes the PI controller, employing nominal values of capacitance (generally producer declared) and PVG dynamic resistance, typically calculated at standard test condition (STC) MPP [21] 


$$
C_{w c}(s)=-\frac{k_{p_{-} w c} s+k_{i_{-} w c}}{s} .
$$

The resulting closed-loop transfer function is dependent on two different conditions, $\mathrm{Bc}$ and worst-case (Wc). Analysing the system from the Wc scenario produces a different group of equations

$$
P_{w c}=-\frac{\left|G_{w c}\right|}{1-s / \omega_{p v_{-} w c}} .
$$

$P_{w c}(17)$ represents the plant when using the Wc position for designing the controller:

$$
\begin{gathered}
c l_{w c}=\frac{C_{w c} P_{w c}}{1+C_{w c} P_{w c}}, \\
c l_{w c}= \\
=\frac{\omega_{p v_{-} w c} G_{w c} k_{i_{-} w c}\left(1+k_{p_{-} w c} / k_{i_{-} w c}\right)}{s^{2}+s\left(G_{w c} k_{p_{-} w c}-1\right) \omega_{p v_{-} w c}+G_{w c} k_{i_{-} w c} \omega_{p v_{-} w c}} .
\end{gathered}
$$

Equation (19) establishes the closed-loop (cl) Wc system, and uses the Wc scenario for development and calculation of controller constants, creating a stable system in all areas of operation. Utilizing (19) and shaping it as a standard form of an underdamped system allows extracting natural frequency and damping ratio. Thus, (20), (21) allows extracting Wc values of natural frequency $(\omega)$ and damping ratio $(\xi)$, respectively, for any given set of variables, and will be used for further investigation:

$$
\begin{gathered}
\omega_{w c}=\sqrt{G_{w c} k_{i_{-} w c} \omega_{p v_{-} w c}}, \\
\xi_{w c}=\frac{\left(G_{w c} k_{p_{-} w c}-1\right) \omega_{p v_{-} w c}}{2 \omega_{w c}} .
\end{gathered}
$$

It should be noted that constants $k_{p}$ and $k_{i}$ are calculated to Wc. Analysis of $\mathrm{Bc}$ will demonstrate the influence of Wc on controller design.

Evaluating the $\mathrm{Bc}$ scenario generates the next group of equations

$$
P_{B c}=-\frac{G_{B c}}{1+s / \omega_{p v \_\mathrm{Bc}}},
$$

with $\mathrm{PBc}$ (22) representing the plant at $\mathrm{Bc}$ operation:

$$
c l=\frac{C_{w c} P_{B c}}{1+C_{w c} P_{B c}} .
$$

This leads to the close-loop (23) while using a Wc controller design

$$
\begin{gathered}
c l_{B C}= \\
=\frac{\omega_{p v_{-} B c} G_{B c} k_{i_{-} w c}}{s^{2}+s\left(G_{B c} k_{p_{-} w c}+1\right) \omega_{p v_{-} B c}+G_{B c} k_{i_{-} w c} \omega_{p v_{-} B c}} .
\end{gathered}
$$

Eventually, the closed-loop receives the Bc component in the form of (24):

$$
\begin{gathered}
\omega_{B c}=\sqrt{G_{B c} k_{i_{-} w c} \omega_{p v_{-} B c}}, \\
\xi_{B c}=\frac{\left(G_{B c} k_{p_{-} w c}-1\right) \omega_{p v_{-} B c}}{2 \omega_{B c}} .
\end{gathered}
$$

Equations (25) and (26) demonstrate the influence of the $\mathrm{Bc}$ operation point, whereas the system uses a controller designed for Wc. The effects of $k_{p}$ and $k_{i}$ constants are clearly shown in the rate of response and restraint of the system. The most noticeable variable between $\mathrm{Wc}$ and $\mathrm{Bc}$ is the apparent value of $\xi$, due to significant gain magnitude and $\omega_{p v}$ variation. Consequently, this leads to different $\xi$ values as shown in Fig. 8, while the second variables are $\mathrm{G}$ and $\omega_{\mathrm{pv}}$ as demonstrated in Fig. 6 and 7, respectively. $\omega_{p v}$ and $\mathrm{G}$ possess significant changes and due to depending on $k_{i_{-} w c}$ values, eventually force the system to behave not as required.

\section{BEHAVIOURAL ANALYSIS}

A design for $\mathrm{Wc}$ due to $v_{\text {bat }}^{\max }$, indicates that while there is no steady state error for step input, transient performance would be heavily influenced by environmental variables and the operating point. For a verification of the receiving transfer function $\operatorname{Pr}$ (13) which does not include all the components of the plant such as Vo and load $\left(v_{B A T}\right.$, $R_{B A T}$ ), see Fig. 5. Using PV values of Table I demonstrates how the Bode curve is performed when using a complete transfer function, including all the components of small signal analysis (blue line). On the other hand, the red line representing the simplified transfer function, does not include all components. Analysis of the Bode diagram reveals that at working frequency, the lines are correlated. Consequently, the system will respond as designed when using reduced transfer function.

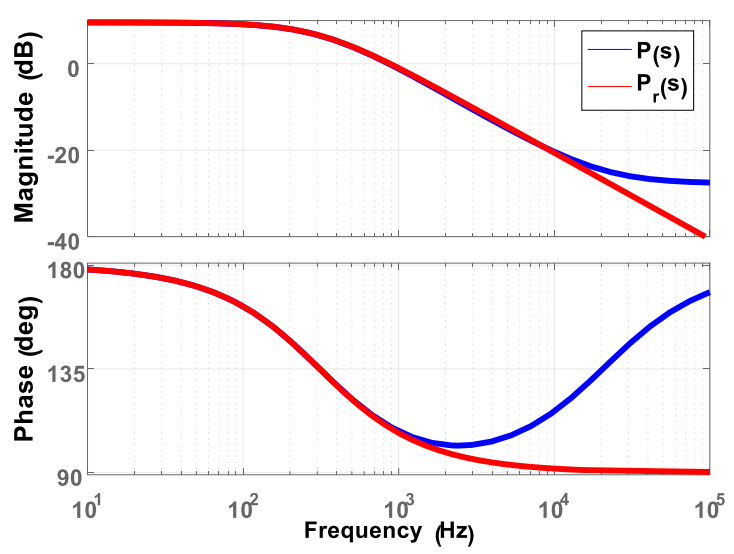

Fig. 5. Bode response of complete and simplified (Pr) plants.

In order to demonstrate the behaviour of the components that significantly influence the control response, a linearization of the PV $\mathrm{i}-\mathrm{v}$ curve is used. The curves are separated into two main sections: 1) the constant current section and 2) the constant voltage section [22]. Each part is 
linearized for simplicity but still provides a good result in imitation of a real $\mathrm{i}-\mathrm{v}$ curve.

With a focus on $\omega_{p v}, \mathrm{G}, \xi, R_{P V}-R_{s}$, in accordance with a Wc control design scenario, which is designed for stability in all conditions, the controller is forced to respond to a variety of working points, while the system is mostly nonfunctional at Wc. Consequently, at a different operating point, the influence of the coefficient in the controller equation (19) on the closed-loop response is incredibly significant. Simulations in Fig. 6-Fig. 9 demonstrate variation in values along the $\mathrm{i}-\mathrm{v}$ curve.

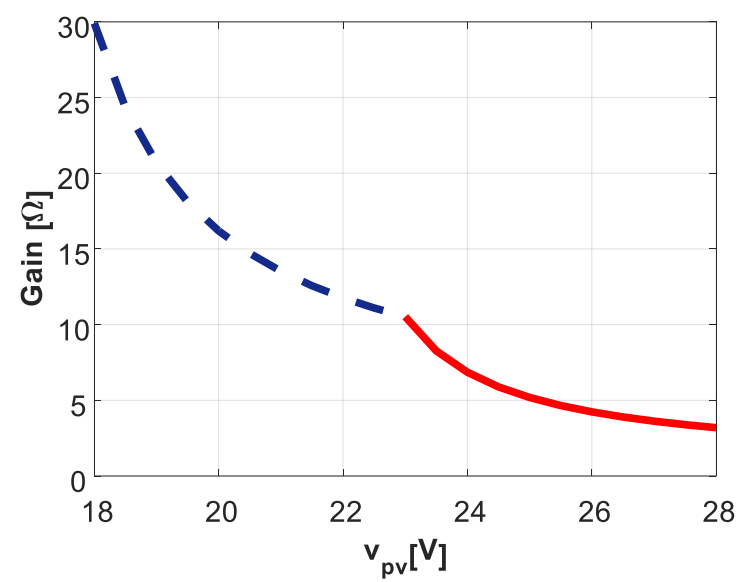

Fig. 6. Gain versus $v_{p v}$.

Figure 6 reveals variation in gain (10) across the entire $\mathrm{PV}$ voltages, which is stirred from almost zero to significant values. As the blue line represents the constant current section and the red line represents the constant voltage section, it is important to note that, crossing point between blue line and red line presenting the MPP point. Hence, it clearly shows the difference in gain behaviour and magnitude before and after the MPP point.

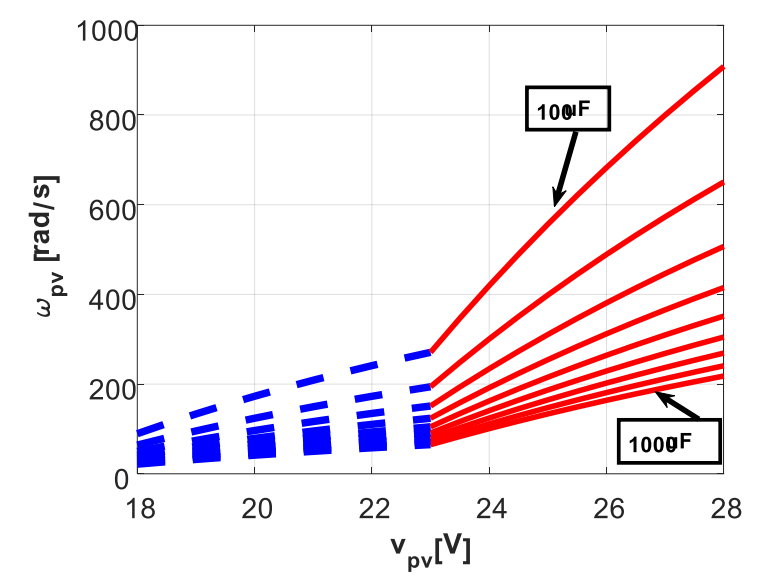

Fig. 7. $\omega_{p v}$ versus $v_{p v}$.

Figure 7 reveals the significant variation of $\omega_{p v}$ (12). In this case, the internal capacitor has an important influence, actualized by each curve, which possesses a different capacitance value and changes from $1000 \mathrm{uF}$ to $100 \mathrm{uF}$. It is clearly shown that as the voltage shifts from MPP where $R_{P V}-R_{s}=0, \omega_{p v}$ increases sharply, influencing the system open-loop response. The effect becomes significant as the capacitor value decreases.
The damping ratio $(\xi)$ plays an important role from the control perspective, predicting performance along the operation points, and, additionally, for different internal capacitors, as shown in Fig. 8. Thus, performance can be assessed in advance and the correct component for the system can be pre-selected. Figure 8 shows the damping ratio increase as voltage increases, meaning that at some point the system will not be fast enough for proper tracking.

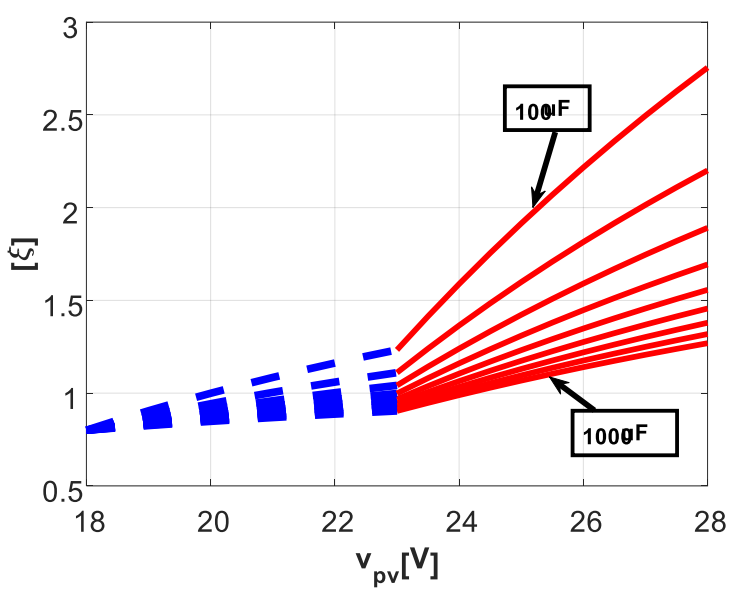

Fig. 8. $\xi$ versus $v_{p v}$.

Since the controller coefficients $k_{p}$ and $k_{i}$ are calculated for Wc, Thus ,the rest of the components in the close-loop transfer function $\left(\omega_{p v} G \xi R_{P V}-R_{S}\right)$ must be modified and adapted to the new condition due to dependence on $R_{P V}-R_{S}$. Figure 9 reveals the significant change of $R_{P V}-R_{S}$ along the operation voltage, the vertical black line representing the chosen voltage for worst-case scenario. It is thus clearly shown that choosing the incorrect voltage can lead to an unstable system.

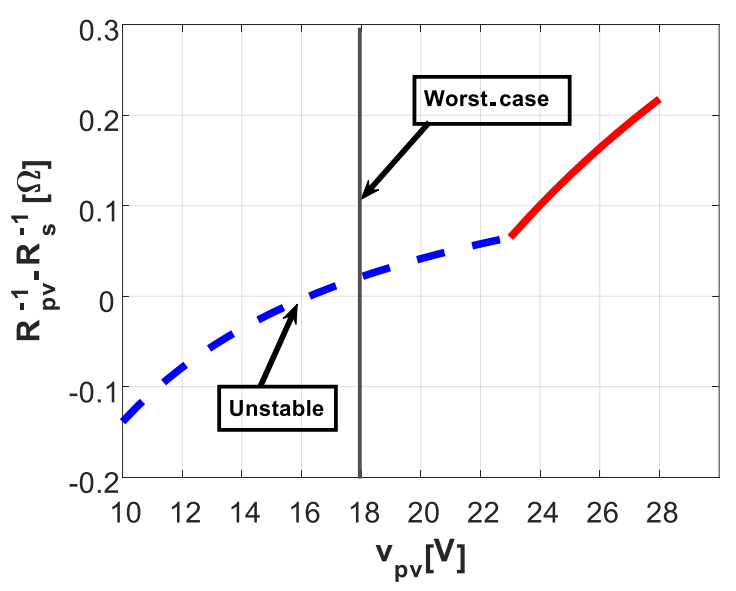

Fig. 9. $R_{P V}^{-1}-R_{s}^{-1}$ versus $v_{p v}$.

Extended system performance evaluation of the control system is demonstrated in Fig. 7, Fig. 8, where the internal capacitor is changed from $1000 \mathrm{uF}$ to $100 \mathrm{uF}$. The variability value influences $\omega_{p v}$ and $\xi$. During the time that the capacitor values decreased, $\omega_{p v}$ accelerates in

Fig. 7 and $\xi$ is increases in Fig. 8. Additionally, each curve which represents a capacitor value also behaves similarly 
according to the voltage value of maximum power point (MPP). Hence, as the voltage value recedes from the MPP to the current source aspect or voltage source aspect its influence is the same. That is, $\omega_{p v}$ grows faster and $\xi$ increases as the voltage changes from $\mathrm{Wc}$ to $\mathrm{Bc}$.

\section{Simulations}

To demonstrate the revealed performance, take into account a $100 \mu \mathrm{F}$ capacitor-terminated BPS-interfaced PVG with STC parameters summarized in Table I. Consider PVG dynamic resistance bounded by $\left(i_{s c} / v_{\min }\right) 0.32 \Omega<R_{P V}$ $<10 \Omega\left(i_{\min } / v_{\max }\right)$. That is, i.e. it may experience almost an exponential increase when the PVG operating point varies from maximum to minimum operation voltage.

TABLE I. PV STC DATA.

\begin{tabular}{|c|c|}
\hline Open circuit voltage & $28 \mathrm{~V}$ \\
\hline Short circuit current & $5.9 \mathrm{~A}$ \\
\hline Maximum power voltage & $23 \mathrm{~V}$ \\
\hline Maximum power current & $5.1 \mathrm{~A}$ \\
\hline Rated power & $117 \mathrm{~W}$ \\
\hline
\end{tabular}

Moreover, assume that input capacitance value may reside in the range of $1000 \mu \mathrm{F}>\mathrm{CI}>100 \mu \mathrm{F}$. Consequently, all possible $\omega_{p v}$ and $\xi$ are already shown in Fig. 7, Fig. 8.

Figure 10 demonstrates tracking simulation results of 1 sun irradiation, operating a PVG voltage loop compensated by a calculating controller (16) for a Wc scenario, with $\omega_{\mathrm{c}}=200 \pi$ $\mathrm{rad} \cdot \mathrm{s}^{-1}, \mathrm{c}_{\mathrm{in}}=100 \mu \mathrm{F}$ and $\mathrm{R}_{\min }=0.32 \Omega$ (STC value). The dashed line indicates system performance when a $50 \mathrm{uF}$ internal capacitor is used. Closed-loop dynamics dependence on the operating point is quite evident. The system is highly over-damped at voltages higher than MPP (high $\xi$ value) and under-damped for voltages lower than MPP (low $\xi$ value). The dashed line indicates the use of a restricted capacitor smaller than the minimum required. By combining Fig. 6-Fig. $9 \mathrm{G}, \omega_{p v}, \xi, R_{P V}-R_{s}$ it is possible to establish the result of the dashed line during the planning stage and can help regulate the correct capacitor for required performance.

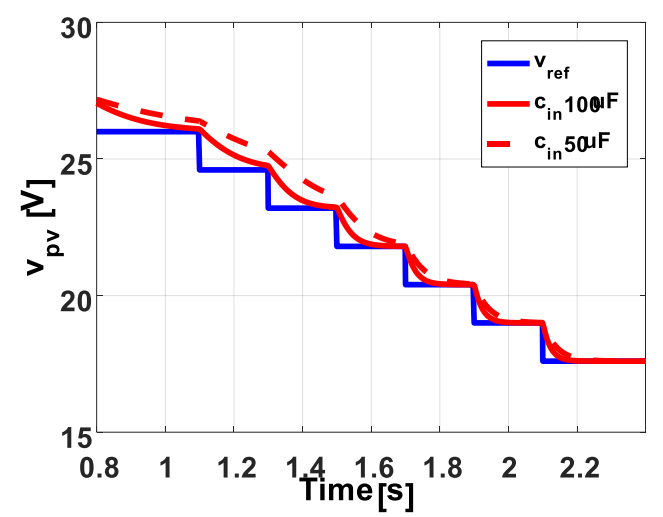

Fig. 10. System response for different internal capacitor.

\section{CONCLUSIONS}

This paper presented a novel mathematical analysis approach for photovoltaic generator. Control strategy of current mode control interfacing a buck DC-DC converter and PVG transfer function as a unified system. Since PVG is characterized by several operation point and dependent environmental-variable nonlinear behaviour the control task nontrivial. The proposed method rewrites the system transfer function and simplified it and the new mathematical equations of unified system plant bounded by worst case scenario are revealed. Hence, the simplified transfer function assists to set the nominal plant without entering an unstable region due the existence of the right-hand pole in buck converter. Moreover, the revealed system behaviour from main components perspective enables the user to preestimate system behaviour for specific conditions or regions of operation, aimed to optimal control design. The new analysis method is essayed by series of simulations that validate the proposed strategy.

\section{REFERENCES}

[1] M. Sitbon, S. Schacham, T. Suntio, A. Kuperman, "Improved adaptive input voltage control of a solar array interfacing current mode controlled boost power stage", Energy Conversion and Management, vol. 98, pp. 369-375, 2015. DOI: 10.1016/j.enconman.2015.03.100.

[2] J. Kivimaki, S. Kolesnik, M. Sitbon, T. Suntio, A. Kuperman, "Design guidelines for multiloop perturbative maximum power point tracking algorithms", IEEE Trans. Power Electronics, vol. 33, pp. 1284-1293, 2018. DOI: 10.1109/TPEL.2017.2683268.

[3] M. Sitbon, S. Lineykin, S. Schacham, T. Suntio, A. Kuperman, "Online dynamic conductance estimation based maximum power point tracking of photovoltaic generators", Energy Conversion and Management, vol. 166, pp. 687-696, 2018. DOI: 10.1016/j.enconman.2018.04.053.

[4] N. Panwar, S. Kaushik, S. Kothari, "Role of renewable energy sources in environmental protection: A review", Renewable and Sustainable Energy Reviews, vol. 15, pp. 1513-24, 2011. DOI: 10.1016/j.rser.2010.11.037.

[5] F. Manzano-Agugliaro, A. Alcayde, F. G. Montoya, A. Zapata-Sierra, C. Gil, "Scientific production of renewable energies worldwide: An overview", Renewable and Sustainable Energy Reviews, vol. 18, pp. 134-143, 2013. DOI: 10.1016/j.rser.2012.10.020.

[6] B. Parida, S. Iniyan, R. Goic, "A review of solar photovoltaic technologies", Renewable and Sustainable Energy Reviews, vol. 15 , no. 3, pp. 1625-1636, 2011. DOI: 10.1016/j.rser.2010.11.032.

[7] T. M. Razykov, C. S. Ferekides, D. Morel, E. Stefanakos, H. S. Ullal, H. M. Upadhyaya, "Solar photovoltaic electricity: Current status and future prospects", Solar Energy, vol. 85, no. 8, pp. 1580-1608, 2011. DOI: 10.1016/j.solener.2010.12.002.

[8] S. Kolesnik, M. Sitbon, S. Gadelovits, T. Suntio, A. Kuperman, "Interfacing renewable energy sources for maximum power transfer Part II: Dynamics", Renewable and Sustainable Energy Reviews, vol. 51, pp. 1771-1783, 2015. DOI: 10.1016/j.rser.2015.04.043.

[9] M. A. Abdullah, A. H. M. Yatim, C. W. Tan, R. Saidur, "A review of maximum power point tracking algorithms for wind energy systems", Renewable and Sustainable Energy Reviews, vol. 16, no. 5, pp. 32203227, 2012.DOI: 10.1016/j.rser.2012.02.016.

[10] V. Salas, E. Olias, A. Barrado, A. Lazaro, "Review of the maximum power point tracking algorithms for stand-alone photovoltaic systems", Solar Energy Materials and Solar Cells, vol. 90, no. 11, pp. 1555-1578, 2006. DOI: 10.1016/j.solmat.2005.10.023.

[11] L. Fangrui, D. Shanxu, L. Fei, L. Bangyin, K. Yong, "A variable step size INC MPPT method for PV systems", IEEE Trans. Ind. Electron. vol. 55, no. 7, pp. 2622-2628, 2008. DOI: 10.1109/TIE.2008.920550.

[12] C. S. Krishnamoorthy, S. Rajeev, Artificial intelligence and expert systems for engineers. Boca Raton, FL, USA: CRC Press, 1996. DOI: 10.1201/9781315137773.

[13] E. Rich, K. Knight, Artificial Intelligence. New York, NY, USA: McGraw-Hill, 1996.

[14] J. Leppaaho, J. Huusari, L. Nousiainen, J. Puukko, T. Suntio, "Dynamic properties and stability assessment of current fed converters in photovoltaic applications", IEEJ Trans. Ind. Appl., vol. 131, no. 8, pp. 976-984, 2011. DOI: 10.1541/ieejias.131.976.

[15] R. A. Kumar, M. S. Suresh, J. Nagaraju, "Effect of solar array capacitance on the performance of switching shunt voltage regulator", IEEE Trans. Power Electronics, vol. 21, no. 2, pp. 543-548, 2006. 
DOI: 10.1109/TPEL.2005.869779.

[16] T. Suntio, J. Viinamaki, J. Jokipii, T. Messo, A. Kuperman, "Dynamic characterization of power electronic interfaces", IEEE Journal of Emerging and Selected Topics in Power Electronics, vol. 2, no. 4, pp. 949-961, 2014. DOI: 10.1109/JESTPE.2014. 2313704.

[17] Z. Zhang, X. Cheng, J. Liu, "An improvement method for extracting five parameters of a solar cell based on Lambert-W function with the current-voltage data", Appl. Mech. Mater., vol. 291, pp. 38-42, 2013. DOI: 10.4028/www.scientific.net/AMM.291-294.38.

[18] M. Sitbon, J Leppaaho, T Suntio, A Kuperman, "Dynamics of photovoltaic-generator-interfacing voltage-controlled buck power stage", Journal of Photovoltaics, vol. 5, no. 2, pp. 633-640, 2015. DOI: 10.1109/JPHOTOV.2014.2379094.

[19] R. M. Corless, G. H. Gonnet, D. E. G. Hare, D. J. Jeffrey, D. E.
Knuth, "On the LambertW function", Adv. Comput. Math., vol. 5, no. 1, pp. 329-359, 1996. DOI: 10.1007/BF02124750.

[20] A. Kuperman, M. Sitbon, S. Gadelovits, M. Averbukh, T. Suntio, "Single-source multi-battery solar charger: analysis and stability issues", Energies, vol. 8, no. 7, pp. 6427-6450, 2015. DOI: $10.3390 / \mathrm{en} 8076427$

[21] M. G. Villalva, T. G. de Siqueira, E. Ruppert, "Voltage regulation of photovoltaic arrays: Small-signal analysis and control design", IET Power Electron., vol. 3, no. 6, pp. 869-880, 2010. DOI: 10.1049/ietpel.2008.0344.

[22] M. G. Villalva, E. Ruppert Filho, "Dynamic analysis of the inputcontrolled buck converter fed by a photovoltaic array", Sba: Controle \& Automação Sociedade Brasileira de Automatica, vol. 19 no. 4, pp. 463-474, 2008. DOI: 10.1590/S010317592008000400009 